\title{
Impact of Different Seed Rates on Yield and Economics of Direct Seeded Rice in Eastern Vidharbha Zone of Maharashtra, India
}

\author{
Usha R. Dongarwar ${ }^{1}$, Nitin Patke ${ }^{2}$, L.N. Dongarwar ${ }^{3}$ and Sumedh R. Kashiwar ${ }^{4 *}$ \\ ${ }^{1}$ Krishi Vigyan Kendra, Bhandara (Sakoli), Maharashtra - 441802, India \\ ${ }^{2}$ Zonal Agricultural Research Station, Sindewahi, Maharashtra - 441222, India \\ ${ }^{3}$ Dr. Panjabrao Deshmukh Krishi Vidhyapeeth, Akola, Maharashtra - 444001, India \\ ${ }^{4}$ Institute of Agriculture, Visva-Bharati, Santiniketan, West Bengal - 731236, India
}

*Corresponding author

\section{A B S T R A C T}

\section{Keywords}

Direct-seeded rice, Seed rate, GMR, NMR, Sye-2001, PKV-HMT

Article Info

Accepted:

04 February 2018

Available Online:

10 March 2018
An experiment was conducted, for three consecutive years to evaluate the effect of seed rates on yield and economic traits in Bold as well as Fine seeded rice variety in terms of Direct-Seeded technique. The experiment was laid out in Factorial Randomized Block design and replicated thrice. Study concluded that Pooled means of seed rates at Sakoli indicated that sowing of $75 \mathrm{~kg}$ seed $\mathrm{ha}^{-1}\left(3458 \mathrm{~kg} \mathrm{ha}^{-1}\right)$ was expressively higher in grain yield but was at par with sowing of $50 \mathrm{~kg}$ seedha ${ }^{-1}$. As well as pooled mean at Sindewahi indicated that Variety Sye-2001 was higher in grain yield $\left(3631 \mathrm{Kg} \mathrm{ha}^{-1}\right)$ of Rice than PKV HMT. The pooled mean of grain yield over three seasons and two locations revealed that variety Sye-2001 was significantly higher in grain yield over PKV HMT Variety. Among seed rates, $75 \mathrm{~kg}$ seedha ${ }^{-1}$ recorded significantly higher grain yield over other seed rates but was at par with $50 \mathrm{~kg}$ seedha ${ }^{-1}$. Interaction effect showed the variety Sye-2001 was significantly highest in grain yield at $75 \mathrm{~kg}$ seedha ${ }^{-1}$ and PKV HMT variety at $50 \mathrm{~kg} \mathrm{ha}^{-1}$. Also the highest GMR, NMR and B:C ratio was recorded in Sye-2001 with $75 \mathrm{~kg}$ seed rate $\mathrm{ha}^{-1}$ and in PKV HMT at $50 \mathrm{~kg} \mathrm{Seedha}^{-1}$.

\section{Introduction}

Seed rate has a great impact on plant density and the competitiveness of the crop stand, tiller, time to maturity and yield. Low plant density and improper sowing method are the most important factors of agronomic constraints for obtaining higher yields and have a positive influence on the yield of rice. Optimum plant density is the primary factor for obtaining higher yield in rice (Sivaesarajah et al., 1995). The increase in plant density increases total plant weight per unit area and decreases the total weight per plant (Yoyock et al., 1979). The number of plants per unit area has an impact on plant architecture, modifies growth and development pattern and effects on the production photosynthesis (Abuzar et al., 2011). The increase in plant density increases the yield up to a limit and thereafter a leveling off or decline in yield (Sivaesarajah et al., 1995). The reason for the reduction in yield is due to the reduction in resources per plant. So the reduction in yield 
will not be compensated by increasing plant number. Direct seeding technique offers a useful option to reduce the limitations of transplanted rice. Direct seeding is being practiced in many developed countries where labour is scarce and expensive (Pingali et al., 1994). Direct-seeded rice occupies $26 \%$ of the total rice area in South Asia (Gupta et al., 2006). Direct seeding of rice avoids puddling, does not need continuous submergence, and thus reduces the overall water demand for rice culture. When rainfall at planting time is highly variable, direct seeding may help reduce the production risk (Singh et al., 2006). Direct seeding can also reduce the risk by avoiding terminal drought that lowers the yield of transplanted rice, especially if the latter is established late due to delayed rainfall. Direct seeding can facilitate crop intensification (Singh et al., 2008). In Vidharbha region of Maharashtra, rice is majorly grown by puddled transplanting method, which is laborious and costly method. The peak period of rice transplanting is in the month of July, which results in labour shortage at the time of transplanting. For this instance, the present study aimed to find out the suitable seed rate for bold and fine seeded rice under drill condition, effect of different seed rates on yield and yield attributing characters of drilled rice and the economics

\section{Materials and Methods}

The study was aimed to investigate the effect of different seed rates on yield and growth traits of bold and fine seeded rice varieties. Study conducted during three rainy (kharif) seasons of 2013-2016 at two locations Krishi Vigyan Kendra, Bhandara (Sakoli), Maharashtra, India and Zonal Agricultural Research Station, Sindewahi, Maharashtra, India. The experimental material comprised of two well-known rice varieties viz., Bold seeded: Sye-2001 ( $\left.\mathrm{V}_{1}\right)$ and Fine seeded: PKVHMT $\left(V_{2}\right)$ with five different seed rate combinations like Sowing of $50 \mathrm{~kg}$ seed ha ${ }^{-1}$
$\left(\mathrm{S}_{1}\right), 75 \mathrm{~kg}$ seed $\mathrm{ha}^{-1}\left(\mathrm{~S}_{2}\right), 100 \mathrm{~kg}$ seed ha ${ }^{-1}$ $\left(\mathrm{S}_{3}\right), 125 \mathrm{~kg}$ seed $\mathrm{ha}^{-1}\left(\mathrm{~S}_{4}\right)$ and $150 \mathrm{~kg}$ seed $\mathrm{ha}^{-1}\left(\mathrm{~S}_{5}\right)$. The experiment was planned in a Factorial Randomized Block Design and replicated thrice. The soil of experimental site was analyzed for initial soil nutrient status (Table 1) and the application of recommended dose of 125:62.5:62.5 kg NPK ha ${ }^{-1}$ was common in all combinations. Date of Sowing and harvesting was strictly followed for consequent three years (Table 2).

\section{Results and Discussion}

\section{Growth traits}

Average results observed in growth traits as influenced by various seed rates on Bold and Fine seeded variety throughout three-year shows, as seed rate increases the plant height, grains panicle ${ }^{-1}$, length of panicle and effective tillers sq. $\mathrm{m}^{-1}$ decreases eventually (Table 3). In term of plant height $\mathrm{V}_{1}$ showed up $94.09 \mathrm{~cm}$, was at its best among entire treatment combinations for three years followed by V1V2S3 attended $90.36 \mathrm{~cm}$ and V1V2S2 was at $89.30 \mathrm{~cm}$. Number of tillers sq. $\mathrm{m}^{-1}$ was recorded highest in $\mathrm{V}_{1}$ (531.73) but the fine seeded variety $V_{2}$ showed 442.73 tillers sq. $\mathrm{m}^{-1}$ (Table 3 ). Some different trends had been noticed like the number of tillers sq. $\mathrm{m}^{-1}$ was increasing as seed rate increases in both varieties. Number of effective tillers sq. $\mathrm{m}^{-1}$ was noticed superior at V1V2S3 (289.17) and V1V2S2 (285.33) in Bold as well as Fine seeded variety. Seed rate of 50 and $75 \mathrm{~kg} \mathrm{ha}^{-1}$ results the average panicle length of $20.95 \mathrm{~cm}$ and $20.25 \mathrm{~cm}$ but $\mathrm{V}_{1}$ showed the highest panicle length of $21.59 \mathrm{~cm}$ in consecutive three years average record (Table 3 ). The fine seeded variety $\mathrm{V}_{2}$ recorded the highest 179.09 grains panicle ${ }^{-1}$ afterward $\mathrm{V}_{1} \mathrm{~V}_{2} \mathrm{~S}_{2}$ and $\mathrm{V}_{1} \mathrm{~V}_{2} \mathrm{~S}_{3}$ were at par to each other throughout the growing seasons. Miller et al., (1991) found that panicle is a key factor that determines and contributes $89 \%$ of differences in yield. These results are in line with those of Kenneth et al., 
(1996) who reported rough rice has gained high yield in the optimum plant stand. $V_{1} V_{2} S_{3}$ and $\mathrm{V}_{1} \mathrm{~V}_{2} \mathrm{~S}_{2}$ showed the significantly highest grain yield sq. $\mathrm{m}^{-1}$ of 389.33 and 377.20 but in terms of variety $V_{1}$ results the high in grain yield. This is in agreement with the studies reported by Mahajan et al., (2004), Hardev et al., (2014) and Rajiv et al., (2013). Basically $\mathrm{V}_{1}$ is Bold seeded variety so it has the high test weight of $25.72 \mathrm{~g}$ and $\mathrm{V}_{2}$ was at $14.34 \mathrm{~g}$. Similar results showing that yield of rice linearly increased with seed rate (density) has been reported by Baloch et al., (2002). The plants at low seed rate have sufficient space and this enables to utilize more nutrients, water and solar radiation for better photosynthesis. Hence, the individual plants performed better. This is in agreement with the studies reported by Baloch et al., (2002), Akbar et al., (2004), Prasad et al., (1999), IRRI (2008), Subbaiah et al., (2002), Gill et al., (2008), Sharma et al., (1992), Mahajan et al., (2006), Dongarwar et al., (2015) and Abou-Khalifa et al., (2014).

\section{Yield traits}

Pooled means of three consecutive years at ZARS Sindewahi location point to bold seeded variety $V_{1}$ - Sye-2001 for highest grain yield of $3631 \mathrm{Kg} \mathrm{ha}^{-1}$ than PKV HMT at 3167 $\mathrm{kg} \mathrm{ha}{ }^{-1} \cdot \mathrm{V}_{1} \mathrm{~V}_{2} \mathrm{~S}_{2}$ (75 $\mathrm{kg} \mathrm{ha}^{-1}$ seed rate) was recorded significantly highest yield over other treatment with $3710 \mathrm{~kg} \mathrm{ha}^{-1}$ of yield (Table 4). Interaction effects between variety and seed rate resulted as significant. $V_{1}$ - Sye-2001 recorded $4162 \mathrm{~kg} \mathrm{ha}^{-1}$ grain yield, which was superior, over all other combinations, and $\mathrm{V}_{2}$ PKV HMT, recorded significantly higher yield, in seed rate $S_{1}-50 \mathrm{~kg}$ seed ha ${ }^{-1}$ of 3710 $\mathrm{kg} \mathrm{ha}^{-1}$ (Table 5). This is in agreement with the studies reported by Zhao et al., (2007), Chauhan et al., (2011), Gill et al., (2006), Phuong et al., (2005), Dongarwar et al., (2015) and Kaun et al., (2014). Pooled means of KVK, Bhandara (Sakoli) location for entire three years indicated that, variety $\mathrm{V}_{1}$ - Sye-
2001 was significantly higher, in grain yield of rice, with $3225 \mathrm{~kg} \mathrm{ha}^{-1}$ of grain yield, Whereas $\mathrm{V}_{2^{-}}$PKV HMT recorded grain yield of 2581 $\mathrm{kg} \mathrm{ha}^{-1}$. Among various seed rates, $\mathrm{V}_{1} \mathrm{~V}_{2} \mathrm{~S}_{2}(75$ $\mathrm{kg}$ seed $\mathrm{ha}^{-1}$ ) showed $3458 \mathrm{~kg} \mathrm{ha}^{-1}$ of grain yield was higher but was at par with sowing of $\mathrm{V}_{1} \mathrm{~V}_{2} \mathrm{~S}_{1}$ (50 kg seed $\left.\mathrm{ha}^{-1}\right)$ with the yield of $3319 \mathrm{~kg} \mathrm{ha}^{-1}$ (Table 6). The seed rate $50 \mathrm{~kg}$ and $75 \mathrm{~kg} \mathrm{ha}^{-1}$ ) were at par with each other and significantly superior over other treatments. Pooled means of interaction of variety and seed rate at KVK, Bhandara (Sakoli) revealed that variety bold seeded $\mathrm{V}_{1^{-}}$ Sye-2001 was higher yield at $S_{2}\left(75 \mathrm{~kg} \mathrm{ha}^{-1}\right)$ and fine seeded PKV HMT recorded best results at $S_{1}\left(50 \mathrm{~kg} \mathrm{ha}^{-1}\right)$ seed rates (Table 7). Zhao et al., (2007), Chauhan et al., (2011), Gill et al., (2006), Phuong et al., (2005) and Kaun et al., (2014) also reported similar results. The results of pooled mean of grain yield over three seasons of both locations revealed that bold seeded $\mathrm{V}_{1}$ - Sye-2001 variety recorded $3578 \mathrm{~kg} \mathrm{ha}^{-1}$ of grain yield, which was expressively higher grain yield over fine seeded $\mathrm{V}_{2}$ - PKV HMT Variety with $2874 \mathrm{~kg} \mathrm{ha}^{-1}$ of yield.

Among different seed rates $\mathrm{V}_{1} \mathrm{~V}_{2} \mathrm{~S}_{2}(75 \mathrm{~kg}$ seed $\mathrm{ha}^{-1}$ ) recorded $3584 \mathrm{~kg} \mathrm{ha}^{-1}$ of grain yield which was significantly higher grain yield, over other seed rates but was at par, with $\mathrm{S}_{1}$ $\left(50 \mathrm{~kg}\right.$ seed ha $\left.{ }^{-1}\right)$ which was with $3485 \mathrm{~kg} \mathrm{ha}^{-1}$ of yield (Table 8). Interaction effect between variety and seed rate revealed that that $V_{1}$ Sye2001 variety recorded $4167 \mathrm{~kg} \mathrm{ha}^{-1}$ of grain yield, which was significantly higher grain yield at $S_{2}-75 \mathrm{~kg}$ seed $\mathrm{ha}^{-1} . \mathrm{V}_{2}$ PKV HMT variety recorded yield of $3483 \mathrm{~kg} \mathrm{ha}^{-1}$ at $\mathrm{S}_{1}$ (50 kg seed $\mathrm{ha}^{-1}$ ) (Table 9). This is in agreement with the studies reported by Kumhar et al., (2016), Payman et al., (2008), Walia et al., (2009), Baloch et al., (2002), Akbar et al., (2004), Prasad et al., (1999), IRRI 2008, Subbaiah et al., (2002), Gill et al., (2008), Sharma et al., (1992), Mahajan et al., (2006) and Abou-Khalifa et al., (2014). 


\begin{tabular}{|c|c|c|c|c|}
\hline \multirow[t]{2}{*}{ Particulars } & \multicolumn{2}{|c|}{ Locations } & \multicolumn{2}{|c|}{ Method used } \\
\hline & ZARS Sindewahi & KVK, Sakoli & & \\
\hline $\mathrm{pH}$ & 7.30 & 7.30 & \multicolumn{2}{|c|}{$p \mathrm{H}$ meter (Piper,1966) } \\
\hline $\mathrm{EC}(\mathrm{dsm}-1)$ & 0.22 & 0.18 & \multicolumn{2}{|c|}{ Conductivity meter (Jackson,1967) } \\
\hline $\begin{array}{c}\text { Organic Carbon } \\
(\%)\end{array}$ & 0.48 & 0.49 & \multicolumn{2}{|c|}{$\begin{array}{l}\text { Walkley and Black method } \\
\text { (Jackson,1967) }\end{array}$} \\
\hline $\begin{array}{c}\text { Available N } \\
\text { kg/ha }\end{array}$ & 221.00 & 234.00 & \multicolumn{2}{|c|}{$\begin{array}{l}\text { Alkaline permanganate method } \\
\text { (Subbiah \& Asija, 1956) }\end{array}$} \\
\hline $\begin{array}{l}\text { Available P2O5 } \\
\text { kg/ha }\end{array}$ & 30.2 & 25.6 & \multicolumn{2}{|c|}{ Olsen's method (Jackson,1967) } \\
\hline $\begin{array}{l}\text { Available K20 } \\
\text { kg/ha }\end{array}$ & 290.00 & 318.00 & \multicolumn{2}{|c|}{$\begin{array}{l}\text { Neutral normal ammonium acetate } \\
\text { method (Jackson,1967) }\end{array}$} \\
\hline \multicolumn{5}{|c|}{$\begin{array}{l}\text { Table.2 Dates of sowing and harvesting at ZARS Sindewahi and KVK, Bhandara (Sakoli), } \\
\text { Maharashtra, India }\end{array}$} \\
\hline \multirow[t]{2}{*}{ Parameter } & \multicolumn{2}{|c|}{ Date of sowing } & \multicolumn{2}{|c|}{ Date of Harvesting } \\
\hline & Sindewahi & Sakoli & Sindewahi & Sakoli \\
\hline First Year & 01.07 .2013 & 08.07 .2013 & 08.11 .2013 & 15.11 .2013 \\
\hline Second Year & 01.07 .2014 & 08.07 .2014 & 28.11 .2014 & 11.11 .2014 \\
\hline Third Year & 01.07 .2015 & 08.07.2015 & 10.11 .2015 & 20.11 .2015 \\
\hline
\end{tabular}

Table.3 Average Ancillary Characters as influenced by different seed rates at ZARS Sindewahi and KVK, Bhandara (Sakoli), Maharashtra, India

\begin{tabular}{|c|c|c|c|c|c|c|c|}
\hline Treatments & $\begin{array}{c}\text { Plant } \\
\text { Height } \\
(\mathrm{cm})\end{array}$ & $\begin{array}{c}\text { No. of } \\
\text { tillers } \\
\text { sq. } \mathrm{m}^{-1}\end{array}$ & $\begin{array}{c}\text { No. of } \\
\text { effective } \\
\text { tillers sq. } \\
\mathrm{m}^{-1}\end{array}$ & $\begin{array}{c}\text { Length } \\
\text { of } \\
\text { panicle } \\
(\mathrm{cm})\end{array}$ & $\begin{array}{c}\text { No. of } \\
\text { grains } \\
\text { per } \\
\text { panicle }\end{array}$ & $\begin{array}{c}\text { Grain } \\
\text { yield } \\
\text { sq.m } \\
(\mathrm{g})\end{array}$ & $\begin{array}{c}\text { Test } \\
\text { weight }\end{array}$ \\
\hline $\mathbf{V}_{1}$ & 91.09 & 531.73 & 275.33 & 21.59 & 114.12 & 371.20 & 25.72 \\
\hline $\mathbf{V}_{2}$ & 84.92 & 442.44 & 236.26 & 19.57 & 179.09 & 303.46 & 14.34 \\
\hline $\mathbf{V}_{\mathbf{1}} \mathbf{V}_{2} \mathbf{S}_{1}$ & 86.20 & 461.67 & 232.67 & 20.25 & 137.43 & 288.33 & 20.13 \\
\hline $\mathbf{V}_{\mathbf{1}} \mathbf{V}_{2} \mathbf{S}_{2}$ & 89.30 & 481.50 & 285.33 & 20.95 & 156.53 & 377.00 & 19.96 \\
\hline $\mathbf{V}_{\mathbf{1}} \mathbf{V}_{2} \mathbf{S}_{3}$ & 90.36 & 469.50 & 289.17 & 21.10 & 159.25 & 389.33 & 20.17 \\
\hline $\mathbf{V}_{\mathbf{1}} \mathbf{V}_{2} \mathbf{S}_{4}$ & 88.25 & 512.33 & 253.50 & 20.63 & 148.65 & 351.50 & 20.19 \\
\hline $\mathbf{V}_{\mathbf{1}} \mathbf{V}_{2} \mathbf{S}_{5}$ & 85.92 & 520.44 & 218.33 & 19.98 & 131.15 & 280.50 & 19.72 \\
\hline
\end{tabular}


Int.J.Curr.Microbiol.App.Sci (2018) 7(3): 32-42

Table.4 Pooled Mean of grain yield of Rice $\left(\mathrm{Kg} \mathrm{ha}^{-1}\right)$ as influenced by various treatments at Sindewahi, Maharashtra, India

\begin{tabular}{|c|c|c|c|c|}
\hline \multirow[t]{2}{*}{ Treatment } & \multicolumn{4}{|c|}{ Grain yield $\mathrm{kg} \mathrm{ha}^{-1}$} \\
\hline & 2013-14 & 2014-15 & 2015-16 & Pooled mean \\
\hline \multicolumn{5}{|c|}{ Main plot : Varieties } \\
\hline $\mathbf{V}_{1}$ & 3441 & 3427 & 4023 & 3631 \\
\hline $\mathbf{V}_{2}$ & 3298 & 2781 & 3422 & 3167 \\
\hline SEm \pm & 110 & 91.0 & 49 & 39 \\
\hline CD@ $9 \%$ & NS & 554 & 298 & 236 \\
\hline CV \% & 12.62 & 11.36 & 8.10 & 6.42 \\
\hline \multicolumn{5}{|l|}{ Sub Plot Seed rates } \\
\hline $\mathbf{V}_{1} \mathbf{V}_{2} \mathbf{S}_{1}$ & 3578 & 3245 & 3968 & 3597 \\
\hline $\mathbf{V}_{1} \mathbf{V}_{2} \mathbf{S}_{2}$ & 3612 & 3392 & 4125 & 3710 \\
\hline $\mathbf{V}_{1} \mathbf{V}_{2} S_{3}$ & 3448 & 3099 & 3697 & 3415 \\
\hline $\mathbf{V}_{1} \mathbf{V}_{2} \mathbf{S}_{4}$ & 3217 & 2991 & 3545 & 3251 \\
\hline $\mathbf{V}_{1} \mathbf{V}_{2} \mathbf{S}_{5}$ & 2994 & 2794 & 3279 & 3023 \\
\hline SEm \pm & 114 & 98 & 119 & 78 \\
\hline CD@ 5\% & 341 & 295 & 356 & 233.0 \\
\hline CV \% & 8.26 & 7.75 & 7.80 & 5.60 \\
\hline \multicolumn{5}{|c|}{ Interaction between Variety $\mathrm{x}$ Seed Rate } \\
\hline SEm \pm & 161 & 139 & 168 & 110 \\
\hline CD@ $5 \%$ & NS & 433 & 522 & 342 \\
\hline $\mathrm{CV} \%$ & 8.26 & 7.75 & 7.80 & 5.60 \\
\hline
\end{tabular}

Table.5 Pooled Interaction effect of Grain yield as influenced by different seed rates at Sindewahi, Maharashtra, India

\begin{tabular}{|c|c|c|c|c|c|c|}
\hline Treatments & $S_{1}$ & $\mathrm{~S}_{2}$ & $\mathrm{~S}_{3}$ & $\mathrm{~S}_{4}$ & $\mathrm{~S}_{5}$ & Mean \\
\hline$\overline{V_{1}}$ & 3484 & 4162 & 3697 & 3514 & 3296 & 3631 \\
\hline $\mathbf{V}_{2}$ & 3710 & 3257 & 3132 & 2988 & 2750 & 3167 \\
\hline Mean & 3597 & 3710 & 3415 & 3251 & 3023 & \\
\hline \multicolumn{3}{|c|}{ SEm \pm} & \multicolumn{4}{|c|}{110} \\
\hline \multicolumn{3}{|c|}{ CD@ $9 \%$} & \multicolumn{4}{|c|}{342} \\
\hline \multicolumn{3}{|c|}{ CV \% } & \multicolumn{4}{|c|}{5.60} \\
\hline
\end{tabular}

Table.6 Pooled Mean of grain yield of Rice $\left(\mathrm{Kg} \mathrm{ha}^{-1}\right)$ as influenced by various treatments at Sakoli, Maharashtra, India

\begin{tabular}{|c|c|c|c|c|}
\hline \multirow[t]{2}{*}{ Treatment } & \multicolumn{4}{|c|}{ Grain yield $\mathrm{kg} \mathrm{ha}^{-1}$} \\
\hline & 2013-14 & 2014-15 & $2015-16$ & Pooled mean \\
\hline \multicolumn{5}{|c|}{ Main plot : Varieties } \\
\hline$V_{1}$ & 3424 & 3275 & 3878 & 3225 \\
\hline $\mathbf{V}_{2}$ & 2436 & 2471 & 2835 & 2581 \\
\hline SEm \pm & 118 & 52 & 61 & 44 \\
\hline CD@ $@ 5 \%$ & 720 & 317 & 370 & 266 \\
\hline CV \% & 15.63 & 7.02 & 7.01 & 5.55 \\
\hline \multicolumn{5}{|l|}{ Sub Plot Seed rates } \\
\hline $\mathbf{V}_{1} \mathbf{V}_{2} S_{1}$ & 3079 & 3226 & 3652 & 3319 \\
\hline $\mathbf{V}_{1} \mathbf{V}_{2} \mathbf{S}_{2}$ & 3322 & 3265 & 3788 & 3458 \\
\hline $\mathbf{V}_{1} \mathbf{V}_{2} S_{3}$ & 2872 & 2810 & 3276 & 2986 \\
\hline $\mathbf{V}_{1} \mathbf{V}_{2} \mathbf{S}_{4}$ & 2710 & 2594 & 3183 & 2829 \\
\hline $\mathbf{V}_{1} \mathbf{V}_{2} S_{5}$ & 2667 & 2471 & 2884 & 2674 \\
\hline SEm \pm & 128 & 139 & 126 & 74 \\
\hline CD@ $9 \%$ & 383 & 416 & 379 & 222 \\
\hline CV \% & 10.67 & 11.82 & 9.22 & 5.95 \\
\hline \multicolumn{5}{|c|}{ I Interaction between Variety x Seed Rate } \\
\hline SEm \pm & 181 & 196 & 179 & 105 \\
\hline CD@ $9 \%$ & 562 & 610 & 556 & 327 \\
\hline $\mathrm{CV} \%$ & 10.67 & 11.82 & 9.22 & 5.95 \\
\hline
\end{tabular}


Table.7 Pooled Interaction effect of Grain yield as influenced by different seed rates at Sakoli, Maharashtra, India

\begin{tabular}{|c|c|c|c|c|c|c|}
\hline Treatments & $\mathrm{S}_{1}$ & $\mathrm{~S}_{2}$ & $\mathrm{~S}_{3}$ & $\mathrm{~S}_{4}$ & $\mathrm{~S}_{5}$ & Mean \\
\hline $\mathrm{V}_{1}$ & 3383 & 4172 & 3515 & 3360 & 3197 & 3525 \\
\hline $\mathrm{V}_{2}$ & 3255 & 2744 & 2456 & 2299 & 2151 & 2581 \\
\hline Mean & 3319 & 3458 & 2986 & 2829 & 2674 & \\
\hline \multicolumn{2}{|c|}{} & & \multicolumn{3}{|c|}{327} \\
\hline CD @ 5\% & & \multicolumn{3}{c}{5.95} \\
\hline
\end{tabular}

Table.8 Pooled mean of grain yield $\left(\mathrm{Kgha}^{-1}\right)$ as influenced by different treatments at ZARS Sindewahi and KVK, Bhandara (Sakoli), Maharashtra, India

\begin{tabular}{|c|c|c|c|}
\hline \multirow[t]{2}{*}{ Treatment } & \multicolumn{3}{|c|}{ Grain yield $\left(\mathrm{Kg} \mathrm{ha}^{-1}\right)$} \\
\hline & Sindewahi & Sakoli & Pooled mean $\left(\mathrm{Kg} \mathrm{ha}^{-1}\right)$ \\
\hline \multicolumn{4}{|c|}{ Main plot : Varieties } \\
\hline $\mathrm{V}_{1}$ & 3631 & 3225 & 3578 \\
\hline $\mathbf{V}_{2}$ & 3167 & 2581 & 2874 \\
\hline SEm \pm & 39 & 44 & 30 \\
\hline CD@ $9 \%$ & 236 & 266 & 183 \\
\hline $\mathrm{CV} \%$ & 4.42 & 5.55 & 3.61 \\
\hline \multicolumn{4}{|c|}{ Sub Plot: Seed rates } \\
\hline $\mathbf{V}_{1} \mathbf{V}_{2} \mathbf{S}_{1}$ & 3597 & 3319 & 3458 \\
\hline $\mathbf{V}_{1} \mathbf{V}_{2} S_{2}$ & 3710 & 3458 & 3584 \\
\hline $\mathbf{V}_{1} \mathbf{V}_{2} S_{3}$ & 3415 & 2986 & 3200 \\
\hline $\mathbf{V}_{1} \mathbf{V}_{2} S_{4}$ & 3251 & 2829 & 3040 \\
\hline $\mathbf{V}_{1} \mathbf{V}_{2} \mathbf{S}_{5}$ & 3023 & 2674 & 2848 \\
\hline SEm \pm & 78 & 74 & 53 \\
\hline CD@ $15 \%$ & 233.0 & 222 & 159 \\
\hline $\mathrm{CV} \%$ & 5.60 & 5.95 & 4.02 \\
\hline \multicolumn{4}{|c|}{ Interaction between Variety x Seed Rate } \\
\hline SEm \pm & 110 & 105 & 75 \\
\hline CD@ $9 \%$ & 342 & 327 & 233 \\
\hline CV \% & 5.60 & 5.95 & 4.02 \\
\hline
\end{tabular}

Table.9 Pooled Interaction effect of Grain yield as influenced by different seed rates at ZARS Sindewahi and KVK, Bhandara (Sakoli), Maharashtra, India

\begin{tabular}{|c|c|c|c|c|c|c|}
\hline Treatments & $\mathrm{S}_{1}$ & $\mathrm{~S}_{2}$ & $\mathrm{~S}_{3}$ & $\mathrm{~S}_{4}$ & $\mathrm{~S}_{5}$ & Mean \\
\hline $\mathbf{V}_{1}$ & 3434 & 4167 & 3606 & 3437 & 3246 & 3578 \\
\hline $\mathrm{V}_{2}$ & 3483 & 3001 & 2794 & 2644 & 2450 & 2874 \\
\hline Mean & 3458 & 3584 & 3200 & 3040 & 2848 & \\
\hline \multicolumn{3}{|c|}{ F Test } & \multicolumn{4}{|c|}{ Sig. } \\
\hline \multicolumn{3}{|c|}{ SEm \pm} & \multicolumn{4}{|c|}{75} \\
\hline \multicolumn{3}{|c|}{ CD@ 0 \% } & \multicolumn{4}{|c|}{233} \\
\hline \multicolumn{3}{|c|}{ CV \% } & \multicolumn{4}{|c|}{4.02} \\
\hline
\end{tabular}


Table.10 Average Cost of cultivation, GMR, NMR and B:C ratio as influenced by different treatments at ZARS Sindewahi and KVK, Bhandara (Sakoli), Maharashtra, India

\begin{tabular}{|c|c|c|c|c|c|}
\hline Treatment & $\begin{array}{c}\text { Pooled Grain } \\
\text { yield }(\mathrm{Q} / \mathrm{ha})\end{array}$ & $\begin{array}{c}\text { Cost of } \\
\text { cultivation }\end{array}$ & $\begin{array}{l}\text { Gross Monetary } \\
\text { Return (Rs/ha) }\end{array}$ & $\begin{array}{l}\text { Net Monetary } \\
\text { Return(Rs/ha) }\end{array}$ & $\begin{array}{l}\mathrm{B}: \mathrm{C} \\
\text { ratio }\end{array}$ \\
\hline \multicolumn{6}{|l|}{ Main plot : Varieties } \\
\hline $\mathbf{V}_{1}$ & 3578 & 33500 & 50092 & 18047 & 1.49 \\
\hline $\mathbf{V}_{2}$ & 2874 & 34000 & 51736 & 18714 & 1.52 \\
\hline F Test & Sig & & Sig & NS & \\
\hline SEm \pm & 30 & & 355 & 493 & \\
\hline CD@ @ & 183 & & 2159 & 3001 & \\
\hline CV \% & 3.61 & & 2.70 & 10.39 & \\
\hline \multicolumn{6}{|l|}{ Sub Plot: Seed rates } \\
\hline $\mathbf{V}_{1} \mathbf{V}_{2} \mathbf{S}_{1}$ & 3458 & 32375 & 55380 & 24271 & 1.71 \\
\hline$V_{1} V_{2} S_{2}$ & 3584 & 33062 & 56175 & 24506 & 1.69 \\
\hline $\mathbf{V}_{1} \mathbf{V}_{2} S_{3}$ & 3200 & 33750 & 50391 & 17850 & 1.49 \\
\hline $\mathbf{V}_{1} \mathbf{V}_{2} \mathbf{S}_{4}$ & 3040 & 34438 & 47849 & 14568 & 1.38 \\
\hline$V_{1} V_{2} S_{5}$ & 2848 & 35125 & 44776 & 10760 & 1.27 \\
\hline F Test & Sig & & Sig & Sig & \\
\hline SEm \pm & 53 & & 890 & 1080 & \\
\hline CD @ 5\% & 159 & & 2667 & 3239 & \\
\hline CV \% & 4.02 & & 4.28 & 14.40 & \\
\hline \multicolumn{6}{|c|}{ Interaction between Variety $\mathrm{x}$ Seed Rate } \\
\hline F Test & Sig. & & Sig & Sig & \\
\hline SEm \pm & 75 & & 1258 & 1528 & \\
\hline CD@ $9 \%$ & 233 & & 3916 & 4756 & \\
\hline CV \% & 4.02 & & 4.28 & 14.40 & \\
\hline
\end{tabular}

Table.11 Interaction effect on GMR as influenced by different seed rates at ZARS Sindewahi and KVK, Bhandara (Sakoli), Maharashtra, India

\begin{tabular}{|c|c|c|c|c|c|c|}
\hline Treatments & $\mathrm{S}_{1}$ & $\mathrm{~S}_{2}$ & $\mathrm{~S}_{3}$ & $\mathrm{~S}_{4}$ & $\mathrm{~S}_{5}$ & Mean \\
\hline$\overline{V_{1}}$ & 48074 & 58341 & 50487 & 48114 & 45451 & 50093 \\
\hline $\mathbf{V}_{2}$ & 62688 & 54011 & 50296 & 47586 & 44103 & 51737 \\
\hline Mean & 55381 & 56176 & 50392 & 47850 & 44777 & \\
\hline \multicolumn{3}{|c|}{ SEm \pm} & \multicolumn{4}{|c|}{1258} \\
\hline \multicolumn{3}{|c|}{ CD@5\% } & \multicolumn{4}{|c|}{3916} \\
\hline \multicolumn{3}{|c|}{ CV \% } & \multicolumn{4}{|c|}{4.28} \\
\hline
\end{tabular}

Table.12 Interaction effect on NMR as influenced by different seed rates at ZARS Sindewahi and KVK, Bhandara (Sakoli), Maharashtra, India

\begin{tabular}{|c|c|c|c|c|c|c|}
\hline Treatments & $\mathrm{S}_{1}$ & $\mathrm{~S}_{2}$ & $\mathrm{~S}_{3}$ & $\mathrm{~S}_{4}$ & $\mathrm{~S}_{5}$ & Mean \\
\hline $\mathrm{V}_{1}$ & 17277 & 27067 & 18558 & 15246 & 12085 & 18047 \\
\hline $\mathbf{V}_{2}$ & 31264 & 21946 & 17143 & 13889 & 9330 & 18714 \\
\hline Mean & 24271 & 24506 & 17850 & 14568 & 10707 & \\
\hline \multicolumn{3}{|c|}{ SEm \pm} & \multicolumn{4}{|c|}{1528} \\
\hline \multicolumn{3}{|c|}{ CD@ 5\% } & \multicolumn{4}{|c|}{4756} \\
\hline \multicolumn{3}{|c|}{$\mathrm{CV} \%$} & \multicolumn{4}{|c|}{14.40} \\
\hline
\end{tabular}




\begin{tabular}{|c|c|}
\hline \multicolumn{2}{|c|}{$\begin{array}{l}\text { Table.13 Treatment wise Cost of cultivation (INR ha }{ }^{-1} \text { ) at ZARS Sindewahi and } \\
\text { KVK, Bhandara (Sakoli), Maharashtra, India }\end{array}$} \\
\hline Treatments & Cost of cultivation (INR ha ${ }^{-1}$ ) \\
\hline $\mathbf{V}_{\mathbf{1}} \mathbf{S}_{1}$ & 32250.00 \\
\hline $\mathbf{V}_{1} \mathbf{S}_{2}$ & 32875.00 \\
\hline $\mathbf{V}_{1} \mathbf{S}_{3}$ & 33500.00 \\
\hline $\mathbf{V}_{1} \mathbf{S}_{4}$ & 34125.00 \\
\hline $\mathbf{V}_{1} \mathbf{S}_{5}$ & 34750.00 \\
\hline $\mathbf{V}_{2} \mathbf{S}_{1}$ & 32500.00 \\
\hline $\mathbf{V}_{2} \mathbf{S}_{2}$ & 33250.00 \\
\hline $\mathbf{V}_{2} \mathbf{S}_{3}$ & 34000.00 \\
\hline $\mathbf{V}_{2} \mathbf{S}_{4}$ & 34750.00 \\
\hline $\mathbf{V}_{2} \mathbf{S}_{5}$ & 35500.00 \\
\hline
\end{tabular}

\section{Economics traits}

Labour saving of Direct Seeded Rice reduces $11.2 \%$ of total production cost as well as Direct Seeded Rice methods have several advantages over transplanting (Singh et al., (2005; Naresh et al., (2010). In addition to higher economic returns, Direct Seeded Rice crops are faster and easier to plant and less labor intensive (Jehangir et al., (2005). Thus, it is necessary to change the cultivation system from transplanting to direct seeded rice (Sanjitha Rani and Jayakiran, 2010).

In terms of Gross monetary return, $\mathrm{V}_{1} \mathrm{~V}_{2} \mathrm{~S}_{2}$ recorded the highest GMR with 56175 INR $\mathrm{ha}^{-1}$, in the same combination Net monetary return was also noticed higher with 24506 INR $\mathrm{ha}^{-1}$ with the B:C Ratio of 1.69 (Table 10). Whereas other combinations were not up to the mark for recommendations. The interaction effect of both locations for GMR, NMR and B: C stated that $\mathrm{V}_{1}$ was best with 58341 INR ha $^{-1}, 27067$ INR ha ${ }^{-1}$ of GMR and NMR respectively only when it is transplanted with the seed rate of $S_{2}-75 \mathrm{~kg}$ $\mathrm{ha}^{-1}$ (Table 11). Effect on fine seeded variety $\mathrm{V}_{2}$ - PKV HMT was high in $\mathrm{V}_{2} \mathrm{~S}_{1}$ combination, which was reported 62688 INR ha ${ }^{-1}, 31264$ INR ha $^{-1}$ of GMR and NMR respectively (Table 12). This is in agreement with the studies reported by Huang et al., (2013), Mehala et al., (2016), Singh et al., (2005), Rao et al., (2007), Naresh et al., (2010), Jagagir et al., (2005), Younas et al., (2016), Awan et al., (2005), Kahloon et al., (2012) and Mazher et al., (2017). The cost of cultivation of entire combinations has shown the normal phenomenal results of cultivars as the seed rate increases the cost of cultivations also increases (Table 13). These results were in accordance to Kumar et al., (2011) reported that labor saving of $86 \%$ and cost saving of $87 \%$ in Direct Seeded Rice compared to manual transplanting.

In paddy, a labor saving of 95-99\% in Direct Seeded Rice was recorded compared to transplanting during three years. Sehrawat $e t$ al., (2010) also observed 13-16\% labor saving in Direct Seeded Rice as compared to manual puddled transplanted rice. Kumar (2011) also recorded similar findings and found higher B: $\mathrm{C}$ ratio in Direct Seeded Rice as compared to transplanted rice. To get the highest grain yield, Gross monetary returns and net monetary returns from drilled rice in Eastern Vidarbha Zone of Maharashtra, $75 \mathrm{~kg}$ seed $\mathrm{ha}^{-1}$ for course varieties and $50 \mathrm{~kg}$ seed rate $\mathrm{ha}^{-1}$ for fine varieties with application of 125 : 62.5: $62.5 \mathrm{~kg} \mathrm{NPK} \mathrm{ha}^{-1}$ is recommended. This is in agreement with the studies reported by 
Husaain et al., (2013), Awan et al., (2005), Kumar et al., (2011), Iqbal et al., (2015), Seharawat et al., (2010), Gangawar et al., (2008) and Sidhu et al., (2014).

\section{References}

Abou Khalifa A A., ELkhoby, W., and Okasha, E. M. 2014. Effect of sowing dates and seed rates on some rice Cultivars. African Journal of Agricultural Research. 9(2): 196201.

Abuzar M.R., Sadozai G.U., Baloch M.S., Shah A.A., Javaid T., and N. Hussain N., Effect of plant population densities on yield of maize, The J. of Ani. and Plant Sci., 21(4), 692-695 (2011).

Akbar N. and Ehsanullah. 2004. Agro-Qualitative Responses of Direct Seeded Fine Rice to Different Seeding Densities, Pak. J. of Agric. Sci., 41, 1-2.

Annual Maharashtra State Rice Workshop Progress Report, 4-5 March, 2014 pp. 12.

Awan, T. H., I. Ali, C. M. Anwar, G. M. Sarwar, C. M. Ahmad, Z. Manzoor and M. Yaqub (2005). Economic effect of different plant establishment techniques on rice production. In Proc. Int. Seminar on Rice Crop, RRI, KSK, Lahore, Punjab- Pakistan. 2-3, October 2005. Pp: 226- 231.

Baloch A.W., Soomro A.M., Javed M.A. and Ahmed M., Optimum plant density for high yield in rice, Asian J. Plant Sci., 1, 25-27 (2002).

Chauhan BS, Singh VP, Kumar A and Johnson DE. 2011. Relations of rice seeding rates to crop and weed growth in aerobic rice. Field Crops Research 121: 105-115.

Dongarwar, U. R., Khedikar, G. R., Kashiwar S. R., and Dongarwar L., 2015, Effect of different Organic Sources Available with Farmers on Paddy (Oryza sativa) in Bhandara District of Maharashtra, Journal of Agricultural Engineering and Food Technology, 2 (2), 142-144.

Gangwar, K.S., Tomar. O.K. and Pandey D.K. 2008. Productivity and economics of transplanted and direct-seeded rice (Oryza sativa)-based cropping systems in Indo-
Gangetic plains. Indian J. Agric. Sci. 78: 655-58.

Gill M.S., Ashwini K. and Pardeep K. 2008. Growth and yield of rice (Oryza sativa) cultivars under various methods and times of sowing. Indian Journal of Agronomy. 51(2): 123-127.

Gill MS, Kumar P and Kumar A. 2006. Growth and yield of direct-seeded rice (Oryza sativa) as influenced by seeding technique and seed rate under irrigated conditions. Indian Journal of Agronomy 51(4): 283287.

Gupta R K, Ladha J K, Singh S, Singh R G, Jat M L, Saharawat Y, Singh V P, Singh S S, Singh G, Sah G, Gathala M, Sharma R K, Gill M S, Alam Murshad, Mujeeb Ur Rehman Hafiz, Singh U P, Mann, Riaz A Pathak, Chauhan H, Bhattacharya B S and Malik P R K. 2006. Production Technology for direct seeded rice. Rice- Wheat Consortium for the Indogangetic Plains, pp 16. New Delhi.

Hardev, R. Singh, J. P. Bohra, J. S. Singh K. R. and Sutaliya, J. M. 2014. Effect of seedling age and plant spacing on growth, yield, nutrient uptake and economics of rice genotypes under system of rice intensification. Indian J. Agron., 59(2): 256-260.

Huang, H.-P., S.-M. Ma, E.-D. Lin, et al., (2013). Benefits comparison analysis of different rice and wheat cropping patterns to adapt to climate change. Adv. Clim. Change Res., 4(3), doi:10.3724/SP.J.1248.2013.182.

Hussain, S., Ramzan, M., Rana, M. A., Mann R. A. and Akhter, M. 2013. Effect of various planting techniques on yield and yield components of rice. The Journal of Animal \& Plant Sciences, 23(2): 672-674.

International Rice Research Institute (IRRI). 2008. Rice Production Training Module: Method of Planting Rice. IRRI, Los Barios, Laguna, Philippines. pp. 1-13.

Iqbal, M. F., Hussain, M., Waqar, M. Q., and Ali, M. A. 2015. Effect of sowing methods on disease of paddy. Int. J. Adv. Mutli-discip. Res. 2(10):4-7.

Jackson, M.L., 1973. Soil Chemical Analysis. Printice Hall Inc. Engiewood Cliffs. N. J. U.S.A. 
Jehangir, W. A., Masih, I., Ahmed, S., Gill, M. A., Ahmad, M., Mann, R. A, Chaudhary, M. R., and Turral, H.2005. Sustaining crop water productivity in rice-wheat systems of South Asia: a case study from Punjab Pakistan. In: Draft Working Paper. Inter. Water Manag. Ins. Lahore, Pakistan.

Kahloon, M.H., M.F. Iqbal, M. Farooq, L. Ali, M. Fiaz and I. Ahmad. 2012. A comparison of conservation technologies and traditional techniques for sowing of wheat. J. Anim. Plant Sci. 3: 827-830.

Kashiwar, S. R., Kumar, D., Dongarwar, U. R., Mondal, B., and Nath, T. 2016. Experiences, challenges and Opportunities of Direct Seeded Rice in Bhandara District of Maharashtra. Journal of Energy Research and Environmental Technology (3) 2: 141145

Kaur, S., and Singh, S. 2014. Influence of crop density on weeds, growth and yield of direct-seeded rice. Indian Journal of Weed Science 46(4): 318-321.

Kenneth and Ronnie S. Halms, Seeding rate effect on rough rice yield, head rice and total milled rice, Agron. J., 88, 82-84 (1996)

Kumar, V. and Ladha, J. K. 2011. Direct seeding of rice: Recent developments and future research needs. Adva. Agro. 111: 297-413.

Mahajan G, Chauhan B S and Johnson D E. 2009. Weed management in northwestern IndoGangetic Plains. Journal of Crop Improvement 23: 366-82.

Mahajan G, Sardana V, Brar AS and Gill MS. 2006. Effect of seed rates, irrigation intervals and weed pressure on productivity of direct-seeded rice (Oryza sativa). Indian Journal of Agricultural Science 76 (12):756-759.

Mahajan, G. Sardana, V. Brar, A. S. and Gill, M. S. 2004. Grain yield comparison among rice (Oryza sativa L.) varieties under direct seeding and transplanting. Haryana $J$. Agron., 20 (1/2):68-70.

Mazher Farid Iqbal, Muzzammil Hussain and Abdul Rasheed. (2017). Direct seeded rice: purely a site specific technology. Int. J. Adv. Res. Biol. Sci. 4(1): 53-57. DOI: http://dx.doi.org/10.22192/ijarbs.2017.04.0 1.006
Mehala Vinay, et al., (2016) Impact of Direct Seeded Rice on Economics of Paddy Crop in Haryana. International Journal of Agriculture Sciences, ISSN: 0975-3710 \& E-ISSN: 0975-9107, Volume 8, Issue 62, pp.-3525-3528.

Miller B.C., Hill J.E. and Roberts S.R., Plant population effects on growth and yield in water seeded rice, Agron. J., 83, 291-297 (1991)

Naresh R.K., Gupta Raj K., Singh B., Kumar Ashok; Shahi U.P., Pal Gajendra;Singh,Adesh; Yadav Ashok Kumar;and Tomar S.S.2010. Assessment of No-Tillage and Direct Seeding Technologies in rice-wheat rotation for Saving of Water and Labor in Western IGP. Progr. Agri. Int. J. 10 (2): 205- 218.

Payman G and Singh S. 2008. Effect of seed rate, spacing and herbicide use on weed management in direct seeded upland rice (Oryza sativa L.) Indian Journal of Weed Science 40 (1\&2): 11-15

Phuong LT, Denich M, Vlek PLG and Balasubramanian V. 2005. Suppressing weeds in direct-seeded lowland rice: Effects of methods and rates of seeding. Journal of Agronomy and Crop Sciences 191:185-194.

Pingali, P.L. and Rosegrant, M.W. 1994. Confronting the environmental consequences of the green revolution. In: Proceedings of the 18th Session of the international Rice Commission, Rome. FAO, Rome, Italy, pp. 59-69

Piper C. S., 1966. Soil and plant analysis. Hans publishers, Bombay. 368.

Prasad, M.K., S.B. Singh, J.M Singh and RP. Sinha. 1999. Effect of seeding method, seed rate and nitrogen splitting on yield attributes of direct seeded rice. Ind. J. App. Bio. 9(1): 55-57.

Rajiv, S. K. 2013. Response of basmati (Oryza sativa L.) rice varieties to system of rice intensification (SRI) and conventional methods of rice cultivation. Ann. Agric. Res., 34 (1): 50-56.

Rao, A.N., Johnson, D.E., Shivaprasad, B., Ladha, J.K. and Mortimer, A.M. 2007. Weed management in direct-seeded rice. Adv. Agro. 93: 153-255. 
Sanjitha Rani T. and Jayakiran K.2010.Evaluation of different planting techniques for economic feasibility in Rice. Elec. J. Envir. Agri. Food Chem. 9 (1):150-153.

Seharawat, Y.S., Bhagat Singh, Malik, R.K., Ladha, J. K., Gathala, M., Jat, M.L. and Kumar, V. 2010. Evaluation of alternative tillage and crop establishment methods in a rice-wheat rotation in North Western IGP. Field Crops Res., 116: 260- 267.

Sharma. A. R. 1992. Effect of varying seed rates and transplanting colonel tillers on the performance of rice under intermediate deep water conditions $(0-80 \mathrm{~cm})$. Journal of Agricultural Science. 119(2): 171- 177

Sidhu, A. S., Kooner, R. and Verma, A. 2014. Onfarm assessment of direct-seeded rice production system under central Punjab conditions. Journal of Crop and Weed, 10(1): 56-60

Singh S, Ladha J K, Gupta R K, Bhushan L and Rao A N. 2008. Weed management in aerobic rice systems under varying establishment methods. Crop Protection, 27: 660-9.

Singh Samar, Sharma R K, Govindra Singh, Singh S S, Singh U P, Gill M A, Jat M L, Sharma S K, Malik R K, Josan A S and Gupta R K. 2005. Direct Seeded Rice: A Promising Resource Conserving Technology. RiceWheat Consortium for the Indo- Gangetic Plains, New Delhi.

Singh Y, Singh G, Johnson D, Mortimer M. 2005. Changing from transplanted rice to direct seeding in the rice-wheat cropping system in India. In: Rice is Life: Scientific Perspectives for the 21 st Century, Tsukuba, Japan: Proceeding of the Word Rice Research Conference, 4-7 November 2004; pp. 198- 201.

Singh, S.P. Sreedevi, B. Kumar, R.M. and Subbaiah, S.V. 2008. Grain yield and economics of wet direct sown rice under different establishment methods and nitrogen schedules. Oryza 45 (3):245-246.

Singh, Y. P. Singh, G. Singh, S. P. Kumar, A. Sharma, G., Singh, M.K. Mortin, M. and Johnson, D. E. 2006. Effect of weed management and crop establishment methods on weed dynamics and grain yield of rice. Indian J. Weed Sci., 38 (1 and 2): 20-24.

Sivaesarajah K., Sangakkara U.R. and Sandanam S., Effect of plant density, nitrogen and gypsum on yield parameters of groundnut (Arachis hypogea L.) in regosols of Batticloa district, Trop. Agric. Res., 7, 112123 (1995)

Subbaiah S. V., Balasubramanian V. and Krishnaiah K. 2002. Evaluation of drum seeder in puddle rice fields. AMA, Agricultural Mechanization in Asia, Africa and Latin America. 33(4): 23-26.

Subbiah, B.V. and Asija, G.L. 1956. A rapid procedure for the estimation of available nitrogen in soil.Curr. Sci. 25: 259.

Walia US, Bhullar MS, Nayyar S and Sidhu AS. 2009. Role of seed rate and herbicides on growth and yield of direct-dry seeded rice. Indian Journal of Weed Science 41 (1 \& 2): 33- 36.

Younas, M., Rehman, M. A., Hussain, A., Ali, L., and Waqar, M. Q. 2016. Economic Comparison of Direct Seeded and Transplanted Rice: Evidences From Adaptive Research Area of Punjab Pakistan.Asian J Agri Biol, 2016, 4(1): 1-7.

Yoyock J.Y., Effects of variety and spacing on growth, development and dry matter distribution in groundnut at 2 locations in Nigeria, Exp. Agric., 15, 339-351 (1979)

Zhao DL, Bastiaans L, Atlin GN and Spiertz JHL. 2007. Interaction of genotypes by management on vegetative growth and weed suppression of aerobic rice. Field Crops Research 100(1): 327-340.

\section{How to cite this article:}

Usha R. Dongarwar, Nitin Patke, L.N. Dongarwar and Sumedh R. Kashiwar. 2018. Impact of Different Seed Rates on Yield and Economics of Direct Seeded Rice in Eastern Vidharbha Zone of Maharashtra, India. Int.J.Curr.Microbiol.App.Sci. 7(03): 32-42. doi: https://doi.org/10.20546/ijcmas.2018.703.004 